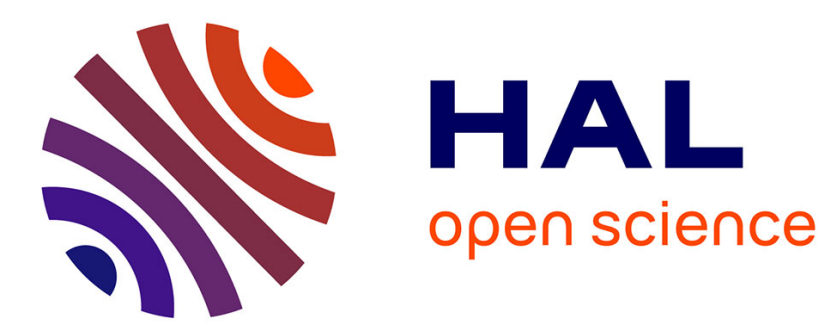

\title{
Liber amicorum du Cornet à dés
}

Patricia Sustrac

\section{- To cite this version:}

Patricia Sustrac. Liber amicorum du Cornet à dés. Les Cahiers Max Jacob, 2017, 17-18, pp.313-331. hal-01806628

\section{HAL Id: hal-01806628 \\ https://hal.science/hal-01806628}

Submitted on 9 Jun 2018

HAL is a multi-disciplinary open access archive for the deposit and dissemination of scientific research documents, whether they are published or not. The documents may come from teaching and research institutions in France or abroad, or from public or private research centers.
L'archive ouverte pluridisciplinaire HAL, est destinée au dépôt et à la diffusion de documents scientifiques de niveau recherche, publiés ou non, émanant des établissements d'enseignement et de recherche français ou étrangers, des laboratoires publics ou privés. 


\section{LIBER AMICORUM DU CORNET À DÉS}

Patricia SUSTRAC*

\footnotetext{
T e 21 mars $1942^{1}$, quelques mois avant sa visite à Saint-Benoît-sur-Loire, Laul Éluard écrivait au « poète pénitent » :
}

Cher Max Jacob,

Vos lettres m'ont été un précieux réconfort. Quel poète peut me faire plus de plaisir que vous! Vous ne savez pas, dans votre retraite, à quel point les jeunes que je vois ici sont d'accord avec moi pour vous rendre justice.

J'ai vos livres, en bel état. Je me permets de vous en envoyer un, pour que vous me le rendiez personnel. Je rêve d'un exemplaire unique. [...]

Voici bien ce qui distingue un exemplaire dédicacé, c'est un exemplaire : « personnel », « unique » pour le collectionneur, l'ami ou le lecteur. Exercice convenu, témoignage d'un lien d'affection, espérance d'une amitié prochaine, les dédicaces sont toujours l'expression d'une main tendue vers un horizon d'idéalité : être lu ou mieux lu, remercier, plaire et s'attacher le lecteur. Jacob n'échappe

* Patricia Sustrac a publié des articles critiques et biographiques et édité plusieurs correspondances de Max Jacob. Elle est Présidente de l'Association des Amis de Max Jacob depuis 2005 et Directrice de publication des cahiers consacrés à l'auteur. 
pas à cet usage général des dédicaces. Cependant, celles de Max Jacob, pour une grande part d'entre elles, échappent aux formules convenues : le poète portait, en effet, beaucoup d'attention à leur rédaction. Marie-Claire Durand Guiziou a montré dans son article pionnier, « À propos d'une dédicace poétique de Max Jacob à André Salmon ", la méticulosité de l'épigraphe imprimée du Terrain Bouchaballe stylistiquement construite dans un jeu de vers libres euphoniques organisée selon un système rythmique complexe et enchâssées dans une subtile combinaison codée 2 . Motivées par l'amitié, l'hommage, la volonté d'être publiées ou de demeurer dans l'intime, les dédicaces s'inscrivent dans un espace littéraire où Jacob exprime ses préférences par des figures esthétiques souvent élaborées et se montre ainsi un redoutable dédicateur. Jacob use de tous les registres du discours : il n'hésite pas à faire l'éloge ou à blâmer son dédicataire (RolandManuel, Philippe de Lavastine) et ne se retient pas de pratiquer l'art de la flatterie.

Nous présentons ici soixante-trois dédicaces qui ornent les trois éditions du Cornet. C'est une collecte restreinte, sans doute, mais si on considère la fragilité des différents recueils vêtus d' " humbles reliures » et fortement soumis aux outrages du temps, c'est une heureuse moisson. La rareté de ces ouvrages et, partant, celle des recueils dédicacés explique d'ailleurs leur prix élevé largement majoré quand la dédicace emprunte à l'iconique et s'enrichit d'un dessin, d'un croquis, d'une gouache ou d'une reliure. Les dédicaces collectées les plus nombreuses sont portées sur l'édition originale. Elles sont majoritairement dédiées à des écrivains, des musiciens, des comédiens ou des poètes. L'édition à compte d'auteur étant restée confidentielle, Jacob a naturellement cherché à faire connaître sa publication auprès de ses pairs et de ses réseaux de sociabilités professionnels. Deux envois sont destinés à des marchands (Basler, Richet). Un autre est porté sur un des exemplaires de tête réservé à l'auteur : destiné à un des souscripteurs du Cornet, il valorise et redouble la valeur de l'ouvrage.

Les dédicaces de Jacob sont motivées par des relations biographiques que l'ajout manuscrit met en scène : le ton y est souvent chaleureux et même enthousiaste, et si la formule d'adresse reste, la plupart du temps, assez convenu (à $\mathrm{X} . .$. , pour $\mathrm{Y} . .$. ), la formule de politesse, quant à elle, est moins codifiée et s'exprime par un lexique plus sensible (« avec ferveur », " sympathiquement »....). La présence de Jacob comme dédicateur est toujours manifeste : elle s'exprime à la première personne (« moi », « je »...), rarement à la troisième personne (« son ami », « Max Jacob qui... »...) et comporte souvent des autocommentaires sur l'œuvre (à Béalu, à Basler). Elles sont toutes signées soit de manière complète soit par ses initiales, souvent datées et localisées : "rue Gabrielle », «à Saint-Benoît ». On notera une domiciliation insolite lorsque Jacob indique la rue 
Gabrielle en offrant son recueil à la Bibliothèque de Quimper en 1939. Lorsque la dédicace est motivée par le don de l'amitié confirmée ou balbutiante, elle bruit des espoirs du rapprochement espéré ou se donne bruyamment comme une vive protestation d'affection perpétuelle (à Auric) quand elle ne s'inscrit pas dans l'infini des mondes (à Sachs). La dédicace peut s'apparenter à une injonction : à Cocteau, Jacob demandera en 1917 - et avec quelle intuition ! - « de faire [s]on monument après [s]a mort. » D'autres intiment des rapprochements littéraires à effectuer par le dédicataire (à Radiguet). Encore certaines ne manquent pas de nous questionner. Que peut bien avoir voulu dire Jacob lorsqu'il admoneste de « se méfier des richesses »? Est-ce une remarque ironique, le rappel à une vertu chrétienne ou une dédicace à clés comme il existe des romans à clés ${ }^{3}$ que seul le dédicataire pourrait décrypter?

On notera dans la collecte quelques belles formules. Tour à tour, Max Jacob se fait moraliste : « La finesse et l'exaltation sont aussi mauvaise conseillère l'une que l'autre » (à $\mathrm{M}^{\mathrm{me}}$ Japy de Beaucourt), directeur de conscience : « Fais un retour vers Dieu Philippe, qui te fera faire des progrès énormes » (à Philippe Lavastine) ou conseiller en élégance en jouant sur le nom du dédicataire : «André/tu piques » (à André Sauvage). Auprès des intimes, Jacob met en abyme le rôle de la dédicace et n'hésite pas à recourir aux calembours : est-il nécessaire, par exemple, d'écrire une dédicace en dédicaçant la dédicace (à Roland-Manuel) ?!

Les dédicaces de Jacob peuvent évoluer vers d'autres fonctions : elles peuvent devenir préface (à Honneger) ou devenir document quand elles donnent des indications sur la genèse des œuvres (à Paul Bonet, à Monny de Boully). À ce titre, elles sont très précieuses pour l'histoire littéraire. Enfin, beaucoup d'entre elles deviennent des textes littéraires à part entière : aphorisme repris dans Visions infernales (à $\mathrm{M}^{\mathrm{me}}$ Japy de Beaucourt) ou poèmes (à Richard Anacréon, à MarieLionèle Floch, à Jean Tuset) : certains seront d'ailleurs repris dans le recueil posthume Derniers poèmes en vers et en prose ou Le Cornet à dés II. Plus insolites sont les dédicaces écrites sous forme épistolaire et qui en empruntent les codes : incipit, adresse, clausule.... (à Pierre Nourry) ${ }^{4}$.

\section{LE CORNET À DÉS : UN EXEMPLAIRE DÉDIÉ}

L'édition définitive du Cornet a été dédiée en 1923 au prince et à la princesse Ghika. Il s'agit du troisième recueil dédié de l'auteur. Le Géant du soleil, conte pour enfants paru en revue ${ }^{5}$, est dédié « À mon cher Daniel »; Histoire du roi Kaboul $1^{e r}$ et du Marmiton Gauwain à « [s]a chère petite cousine Thérèse 
Gompel. » Si l'on écarte l'épigraphe du Géant dont le dédicataire n'est toujours pas identifié, celle du Cornet rejoint le genre des hommages à des mécènes : Thérèse Gompel, outre son lien de parenté avec l'auteur, était en effet la fille de Gustave Gompel, collectionneur fortuné et mécène (prudent) du poète. Si la dédicace de Kaboul manifeste des liens biographiques affectueux incontestables, celle que Jacob adresse aux Ghika révèle plutôt un effet de distance. Les termes sont plus convenus ; seul l'adjectif de clausule vient relever la formule qui paraît celle d'un obligé : «Avec l'hommage respectueux de mon amitié fervente. » Jacob était fréquemment reçu chez les princes à Paris comme à Roscoff. En 1923, il séjournera au Clos Marie d'août à septembre. La dédicace est offerte à la princesse Ghika pour son anniversaire le 11 août $^{6}$, Jacob est encore l'hôte idéal qui prie et médite, et travaille. Mais, ces vacances finistériennes tournèrent rapidement à l'aigre. Malade, indisposé par la présence de nombreux hôtes, irascible, nerveux, provoquant des esclandres, Jacob n'eut d'autre recours que la fuite. Un petit pâté de Chartres, un bonnet et une écharpe rouge scelleront les dons et contre-dons d'une réconciliation mutuelle espérée par les deux camps et délicatement acquise au début de 1924. Sa respectueuse fidélité sera cependant inscrite au fronton de son recueil le plus célèbre que le séjour agité de l'été avait toutefois contribué à parfaire.

Les dédicaces collectées sont présentées par ordre alphabétique et par exemplaire de parution. Elles sont souvent accompagnées de dessins : nous les signalons sans toutefois pouvoir les reproduire tous pour des questions de droits. Placées en page de garde ou de faux-titre, exceptionnellement sur la page d'avanttitre ou la couverture de l'ouvrage (à Francis Dumont), nous avons indiqué leur emplacement mais sans préciser, à chaque fois, le « $\mathrm{J}$ » du patronyme de l'auteur qui indique par sa longueur démesurée l'intensité de l'amitié de Jacob - cette originalité étant d'usage pour la plupart d'entre d'elles. Sauf exceptions, nous avons retranscrit les envois tels qu'ils se présentent au manuscrit : Jacob débute très rarement son envoi par une majuscule ; nous avons suivi cette originalité. Nous avons pensé utile de signaler quelques envois cités par les dédicataires alors que les éditions n'ont pas été indiquées. Les dédicaces ont été patiemment relevées sur des catalogues de ventes, des recueils conservés en collections publiques ou privées. Ce travail a été enrichi par la généreuse compréhension des collectionneurs, des bibliophiles, des libraires, des bibliothécaires. Bien entendu, nous avons puisé dans les recueils ou les épistolaires des dédicataires eux-mêmes. Que chacun trouve ici l'expression de mes remerciements les plus chaleureux. 


\title{
ÉDITION ORIGINALE
}

\section{- Exemplaires SUR Vieux Japon}

À Paul Éluard ${ }^{7}$

Signature autographe de Max Jacob à la justification du tirage avec mention de sa main «Exemplaire $n^{\circ} 5$ ». L'eau-forte de Picasso porte sa signature autographe au crayon.

Marginalia : onze poèmes ou fragments de texte dont voici les incipit : « L'artiste, le mage et le prêtre », "Je suis habillé parce que j'attends des visiteurs », «Quel voyage ! la voiture dans les arbres!», « La mère du prêtre a des pensées intimes », « Pointu est le clocher, le peuplier est pointu », « Je m'envole au-dessus des trottoirs », « La villa en briques humides », " Le président de la République est étonné que la salle soit vide ou presque », "L'Automobile du cardinal ! c'est un sleeping ou salon beige », « Entre deux rochers, la cascade », «Traduire! mais c'est l'auteur qui ne comprends plus ».

\section{À Madame Japy de Beaucourt ${ }^{8}$}

Mes grelots. Maigres lots! ce sont ceux du péché. //à la très belle / très spirituelle et très bonne Madame Japy de Beauco[u]rt/son ami Max Jacob.

\section{- Exemplaires sur Hollande}

\section{À Paul Bonet ${ }^{9}$}

À mon ami Paul Bonet

Petit historique du Cornet à dés

\begin{abstract}
$I$
Oh quel titre! disait Miss Hastings (dame anglaise et femme de Modigliani). En Angleterre, vous savez on vous le volera avant la parution du livre!

On a volé bien autre chose! les poèmes étaient bien connus! on venait le matin 7 rue Ravignan lire le poème de la nuit... les voisins... Picasso, Salmon, Mac Orlan etc.... ce qu'on tapera là-dedans! disait Mac Orlan. En effet, quelqu'un que je ne nommerai pas quand il fut question d'une édition se hâta de faire sous un autre titre (nous ne sommes pas à Londres tout de même) un recueil qu'il voulait être un pastiche et ne réussit pas à l'être. Triomphe des copains! " enfoncé Max!" Et moi à Picasso : "c'est vrai que X... c'est mieux que moi ?- Tu sais bien que les imitations c'est toujours mieux que les inventeurs! » Cela ménage le chou, la chèvre et la vérité. N'empêche que le livre de X”. est tombé dans l'oubli et que le petit Cornet vit encore. Il connut deux éditions Stock (petits livres jaunes) après celle qui fut faite par souscription et chez l'auteur rue Gabrielle, 17, XVIII'.
\end{abstract}


Je me souviens de la lettre d'Albert Thibaudet alors soldat au ministère de la guerre : "Il me semble que tous les dossiers sont pêle-mêle tombés sur mon bureau. " Laurent Tailhade, alerté par un copain, daigna remarquer « Dahlias, Dahlias, que Dalila lia! „J'eus tout de même pas mal de succès.

T.S.V.P//

$J$ 'ai toujours fait du poème en prose ou moitié prose quand mes cinq frères $\mathrm{L}_{e t}$ $\mathrm{moi}^{\perp}$ tout petits revenions de la foire des saltimbanques à la nuit sous la conduite de la bonne. Nous avions très peur dans l'escalier sans minuterie et j'avais improvisé ceci :

" Messieurs les chats et messieurs les voleurs, s'il y a des chats et s'il y a des voleurs, messieurs les chats ne me griffez pas! messieurs les voleurs ne me faites pas peur »

C'est bien le Cornet déjà... Avais-je douze ou quinze ans?...

Plus tard étudiant à Paris, je fréquentais de gros cousins riches et j'ai encore fais ceci :

" Mon pardessus est mon bouclier, mon parapluie est mon défenseur. J’ai gagné 0f50 centimes sur mes ennemis? et vous mademoiselle vous savez danser?»

J'ignorais complètement Jarry et le père Ubu. Il n'était d'ailleurs pas question de littérature encore dans ma vie. Plus tard, après déjà des aventures, me voici employé boulevard Voltaire 137 et en possession d'une jeune dame. Nous habitions 33 boulevard Barbès, je lui dis un jour :

«Elle est si lasse que les paupières des renoncules se ferment sur son chapeau »

Ce n'est que plus tard encore quand il a été avéré que j’étais parmi les poètes et cité dans la fameuse "Après-midi des poètes", conférence faite par Apollinaire aux Indépendants en 1907. (J'exagère, il y avait longtemps que je collectionnais les poèmes en prose) que je me suis appliqué à saisir en moi de toutes manières les données de l'inconscient : mots en liberté, association hasardeuses des idées, rêves de la nuit et du jour, hallucinations etc...

Voir épilogue page $134 / /$

M. $J$.

Épilogue du Petit historique

"Pourquoi ne fais-tu pas une suite au Cornet à dés " me demandait le comte François de Gouy d'Arcy. "C'est le seul homme qui sache ce que c'est que la 
peinture 》 disait de lui Picasso. "Fais-en une pour moi! "Je m'y suis mis et un jour dans le téléphone j'annonçais que j’avais 60 pages : "Viens dîner ! et apporte les 60 pages! » On les lut avec enthousiasme! on téléphonait aux amis et à chaque nouvel arrivant, il fallait les relire. Après minuit on me fit reconduire par le chauffeur rue Gabrielle 17, les bras pleins de fleurs. Le moindre ducaton aurait mieux fait mon affaire.

À quelques temps de là, François me téléphona que son ami Greeley et lui avaient découvert un coin près de Versailles d'où l'on voyait tout Paris : "Viens déjeuner, nous te montrerons ça!"Nous voici donc à contempler Paris près de Versailles : "Et cette atmosphère argentée !...- Oui, dis-je, à Paris il n’y a plus que l'atmosphère qui soit argentée! » Cette allusion fut comprise, François se mit à se plaindre de sa pauvreté et des héritages subtilisés etc. Le soir vint, puis la nuit. Ceci se passait dans un hôtel près de l'Étoile. "Quelle heure estil ? dit François - deux heures à cette belle montre ancienne - elle te plaît cette montre? prends-là ! " J'ai porté cette montre plus de dix ans aux jours de fête et de cérémonies: je lui avais adjoins une longue chaîne en or.

Aujourd'hui je songe à la vendre! à quoi bon quand on vit en ermite posséder des bijoux? (c'est comme mon émeraude). Il est question de 15000 francs, somme qui durera bien autant que ma vie finissante, et allègera certains petits soucis. Jamais le Cornet à dés ne m'aura rapporté lui-même autant que son supplément. Filibuth est de la partie car mon futur acheteur veut l'avoir en souvenir de ce roman dont le sous-titre est : Filibuth ou la montre en or.

\section{- EXeMPlaires ORDinaIres}

\section{À Richard Anacréon ${ }^{10}$}

Cornet à dés... adde/ Le Singe et le Forçat/ «Vous vendez toutes les fourrures? I Toutes sauf le singe /- pourquoi pas le singe /- le patron dit que le singe lui rappelle la Guyane et le temps où il était forçat. »//

Les Chats et la musique/Quand j'étais musicien militaire et qu'on jouait un/certain air. Les gens des cafés, aux terrasses, rapprochaient/leurs chaises les unes des autres comme pour mieux entendre / en se tenant serrés. //

Rédemption/Un ange par un rêve m'a révélé le sort de mes/amis après leur mort. Ne nommons personne et ne/répétons rien! Quant à moi dans ce rêve, j'étais/menuisier et toutes les planches que je touchais/s'enflammaient par un bout. De grands anges/maigres éteignaient le feu/ Max Jacob

\section{À Georges Auric ${ }^{11}$}

Troisième jour du printemps 1918. Max Jacob, 17, rue Gabrielle... P. S. Par une dédicace attention (le mot est d'Auric) ma signature imite ici celle du maître Massenet (12 centimètres). 


\section{À Adolphe Basler ${ }^{12}$}

à Basler / sans espoir / que mon / livre / triomphe / de / son goût sévère / Max Jacob

\section{À Marcel Béalu ${ }^{13}$}

Au très aimé Marcel/après la lecture du Goëland/bravo. M. J. Juillet 1943

\section{À la Bibliothèque de Quimper ${ }^{14}$}

à la Bibliothèque de Quimper. Max Jacob 39

\section{À la Bibliothèque de Quimper ${ }^{15}$}

à la bibliothèque de Quimper. Exemplaire appartenant à Max Jacob, 17 rue Gabrielle, Paris XVIII ime

\section{À Monny de Boully ${ }^{16}$}

Cet exemplaire appartient/à Max Jacob//Je donne cet exemplaire uniquelà Monny de Boully/pour que jamais il ne le vende/ Max Jacob.

\section{À Marguerite Cæatani ${ }^{17}$}

à Marguerite, princesse de Bassiano/Aujourd'hui les bourgeons sur les/branches, et dans ce livre les/les prémices d'un poète. LE CORNET À DÉS qu'avec le plus grand/respect il vous offre comme / un humble hommage/ Max Jacob

\section{À Jean-Ernest Charles ${ }^{18}$}

à un écrivain néocratique, au poète en prose. / Respectueusement.

\section{À Jean Cocteau ${ }^{19}$}

À mon cher Jean/poète admirable. / Moraliste austère/Et auteur dramatique méconnu de soi / même et de mon cher ami/ Max Jacob

\section{À Léon Deffoux ${ }^{20}$}

À Léon Deffoux/enthousiaste et bon avec l'admiration/de son ami déjà ancien. / Max Jacob/le 19 février 1918

\section{À Georges Desse ${ }^{21}$}

à mon cher Georges / poète et savant / que j'aime profondément / ce livre très rare et introuvable / Max Jacob/boiteux, janvier 1930

\section{À Jacques Dyssord ${ }^{22}$}

À mon ami Jacques Dyssord en réponse à de beaux vers. C. Max Jacob 17 juill. 1918 


\section{À Louis Émiée ${ }^{3}$}

à Louis Émiél, son ami/, Max Jacob/Le poète enchaîné sur les bords/de la Garonne veut / et ne veut pas changer de prison/- NB/- Ça vous plaît? - non - moi non plus...

\section{À Léon Eyrolles ${ }^{24}$}

Je ne sais quel sera ton rôle / délicat et fier Eyrolles / dans notre salope de vie / mais je ne crains que tu dévies / tant et si bien je te devine / Max Jacob

\section{À Georges Gabory ${ }^{25}$}

Toi qui a la figure d'un ange / Puisses-tu en avoir la voix ! / Max Jacob/ Premier jour du printemps de 1918

\section{À Florence Gould ${ }^{26}$}

J'ai de la dédicace pour vous dans le cæur.

\section{À Jean Marchat ${ }^{27}$}

La finesse et l'exaltation / sont aussi / mauvaise conseillère / l'une que l'autre / Max Jacob/à Jean Marchat 1933/qu'on aimera jamais autant qu'il mérite de l'être/M. J.

\section{À Arthur Honegger ${ }^{28}$}

à Honegger/qui me demande d'écrire un/mot sur cette page/son ami/Max Jacob/Maintenant une vraie/dédicace./Au musicien Honegger/qui par la magie de ses/accords et par la finesse delson esprit etc... etc.../ Sympathiquement / Max Jacob

\section{À Arthur Honneger}

à Honegger / le poète des sons. / Affectueusement. / Max Jacob/T. S. V. P. //

à Honegger / l'ange rebelle / comme Gabory est le bel ange / Max Jacob

\section{À Georges Hugnet ${ }^{29}$}

J'ai de la dédicace pour toi George Hugnet dans le cœur, le mien est dans ce livre

\section{À Jean Hugo ${ }^{30}$}

Vous êtes le seul peintre qui m'enchante / la peinture est si ennuyeuse! / et l'art... l'art... l'art ... mais vous !/là Jean Hugo/Max/P. S. Je voudrais vous dire quelque choselde très bien et je ne peux pas...jel ne sais pas... Je vous enverrai ce que j'ai / de plus précieux après mon cour et l avec lui. // 


\section{À André Mignot ${ }^{31}$}

à mon cher Mignot que je tutoie avec plaisir et facilitél pour sa fille quand elle aura vingt ans. Max Jacob

\section{À Conrad Moricand ${ }^{32}$}

à mon bien aimé Conrad Moricand. Je te dédie ce portrait de moi-même tel qu'en ce lieu le peignit Picasso le teint rougeau du paysan sot, l'air emprunté et le front blême/rien de commun avec le monsieur bien qui partagera ton repas ce matin. 1933

\section{À Joseph Pérard ${ }^{33}$}

Il y avait une fois un type qui s'appelait Joseph Pérard; tout le monde l'aimait. On fit un concours au dynamomètre pour savoir qui l'aimait le plus. Qu'on approche le dynamomètre du vieil homme de lettres de St-Benoît, le dynamomètre éclate.

\section{À Adolphe Pouthier ${ }^{34}$}

à Adolphe Pouthier, monseigneur l'évêque apostolique d'Orléans (Loiret) len témoignage du respect et de la fidèle affection. / Son diocésain très humble et serviteur dévoué. / Max Jacob/ de Saint-Benoît-s / Loire

\section{À Raymond Radiguet ${ }^{35}$}

a Hef fred Richeds

S'llaime les vocalides

it la flute

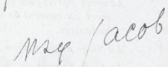

Le Cornet à Dés à Raymond Radiguet / cf ma dédicace du Phanérog [ame]. Max Jacob

\section{À Alfred Richet ${ }^{36}$}

à Alfred Richet /s'il aime les vocalises / et la flûte Max Jacob

\section{À Roland-Manuel ${ }^{37}$}

pourquoi les dédicaces? Si tu sais/que je t'aime, pourquoi le répéter et/si tu en doutes, je le dirai en vain. / Max Jacob

\section{À Mademoiselle Sondaz ${ }^{38}$}

à Mademoiselle Sondaz./En souvenir des mauvais jours / Max Jacob

\section{À Gérard Magistry ${ }^{39}$}

à Gérard M. I La cathédrale de Reims est la/première, Chartres ne vient /qu'en quatrième. / Bourges est faite à la main/d'un seul morceau. / Chartres c'est le marché aux puces. / Quelle méchanceté ! dit Gérard. IJe suis crucifié, ajoute-til. / Max Jacob. // La couronne impériale n'est plus qu'une cage / à serins. M. J. / Et comme cravate, la Tour / Eiffel. / M. J. 


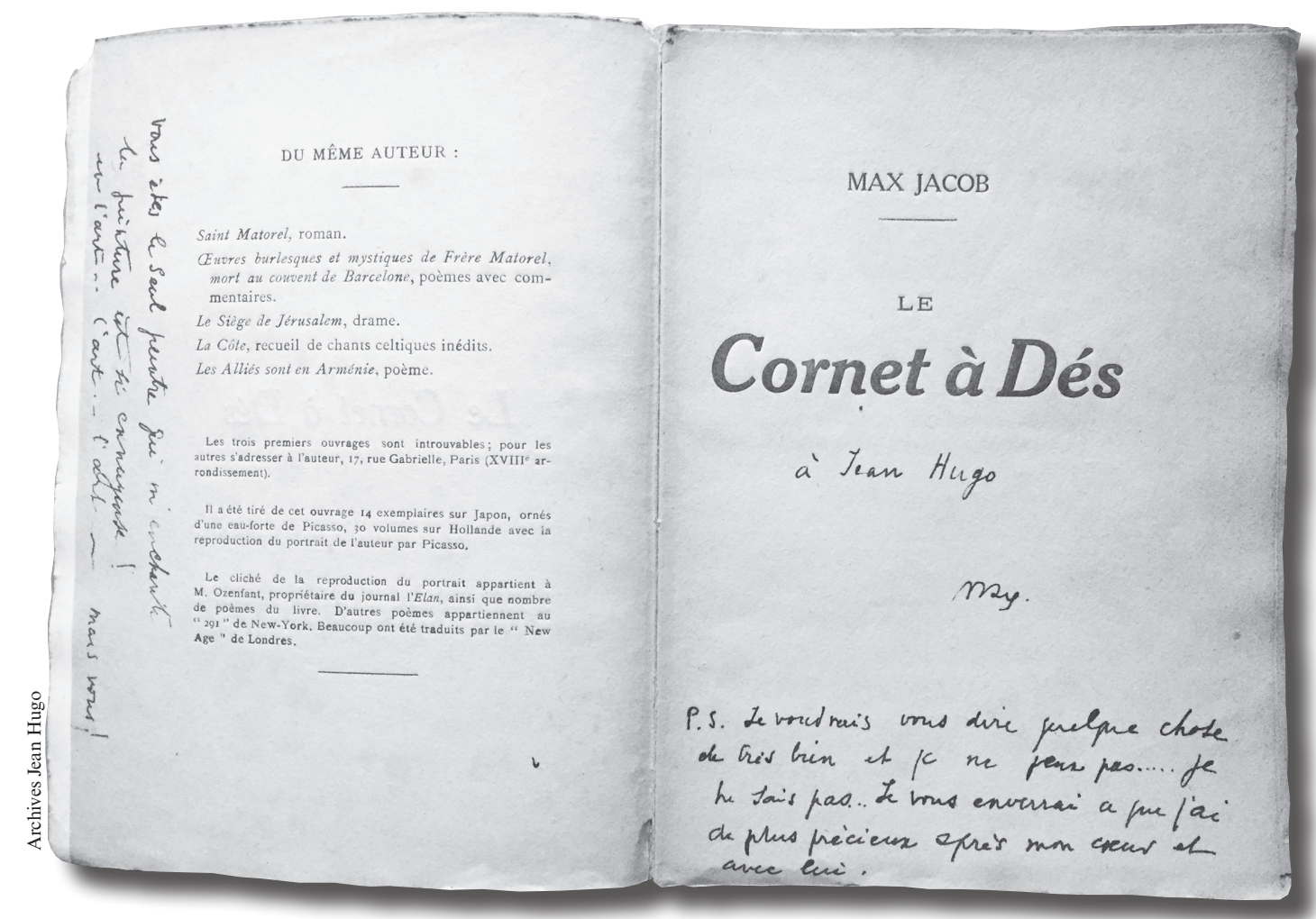

Le Cornet à dés, dédicace à Jean Hugo.

Page de faux-titre verso : «Vous êtes le seul peintre qui m'enchante/la peinture est si ennuyeuse !/ et l'art... l'art... l'art ... mais vous ! // Page de titre : « à Jean Hugo/Max/ P. S. Je voudrais vous dire quelque chose/ de très bien et je ne peux pas... je/ne sais pas... Je vous enverrai ce que j'ai/ de plus précieux après mon cœur et/ avec lui. » 


\section{À un dédicataire non identifié}

Méfiez-vous de la richesse. / Max Jacob

\section{ÉDITION DE 1922}

\section{À Marcel Béalu ${ }^{40}$}

Je n'ai jamais lu un livre plus stupide... J'ai honte... J'ai honte !/ Ceux qui le méprisaient avaient raison, mais pourquoi l'ont-ils imité?

\section{À la Bibliothèque de Quimper ${ }^{41}$}

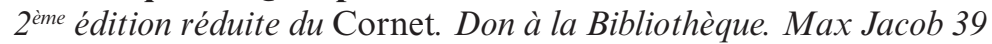

\section{À Francis Dumont ${ }^{42}$}

à Francis Dumont qui essaie de ressusciter Lazare ; avec les remerciements de Lazare

\section{À Paul Méral ${ }^{43}$}

à Paul Méral, poète intense et natif, son ami

\section{À Roland Saucier ${ }^{44}$}

à mon ami Roland qu'il agite les dés et qu'il gagne. Max Jacob. Saint-Benoîtsur-Loire

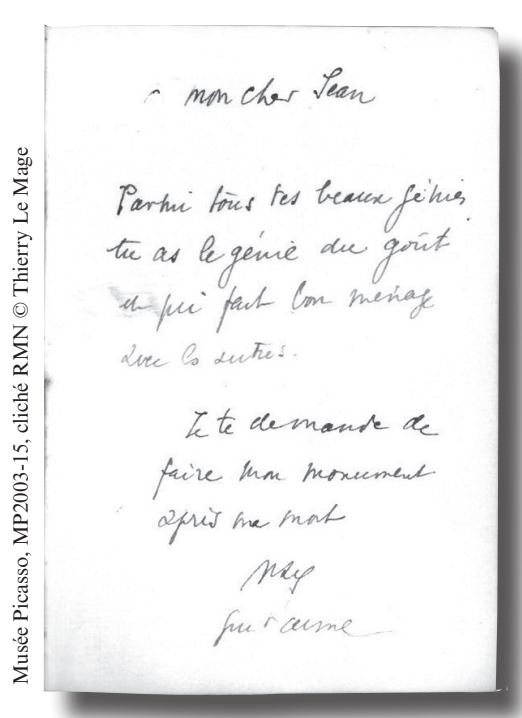

Exemplaire du Cornet à dés dédicacé à Jean Cocteau, éd. 1923, ex. sur Hollande $n^{\circ} 4$
À André Sauvage ${ }^{45}$ Andrél tu piques / Max

À Willy Michel ${ }^{46}$

Puisse le second joueur des/dés être mon ami Willy / Michel/ Max Jacob / avril 36

\section{ÉDITION DE 1923}

\section{- Exemplaires sur Hollande}

\section{À Jean Cocteau ${ }^{47}$}

à mon cher Jean / Parmi tous les beaux génies / tu as le génie du goût let qui fait bon ménage avec les autres. I Je te demande delfaire mon monument / après ma mort / Max / qui t'aime. 


\section{À Paul Éluard ${ }^{48}$}

"Au sujet de moi"/En séparant mon corps de mon âmelpar la pensée de la mort, je les/annonce! en les annonçant, je les/appelle! en les appelant je les crée. / MAX JACOB/à Paul Eluard I que je n'ai pas connu assezl tôt et lors de ma formation/ de ma déformation/Max Jacob/Octobre 42

\section{À Gérard Magistry ${ }^{49}$}

à Gérard / à son gros brouillard doré / à son gros cœur tendre et humble / à sa fine intelligence poétique/scientifiquelà son bel avenir mérité/son ami / Max/St-Benoîts/-Loire 1926. 21 juin.

\section{À Pierre Nourry ${ }^{50}$}

Bien cordialement/À Monsieur Pierre Nourry/En le remerciant de sa sympathiel trop respectueuse. Au respect, je préfère l'amitiélet au piédestaux la fraternité exquise. I Quant au 2 juin, je salue en luilbien de l'intelligence applicable aux sciences/ comme aux arts, intelligence qui voltigelavec grâce

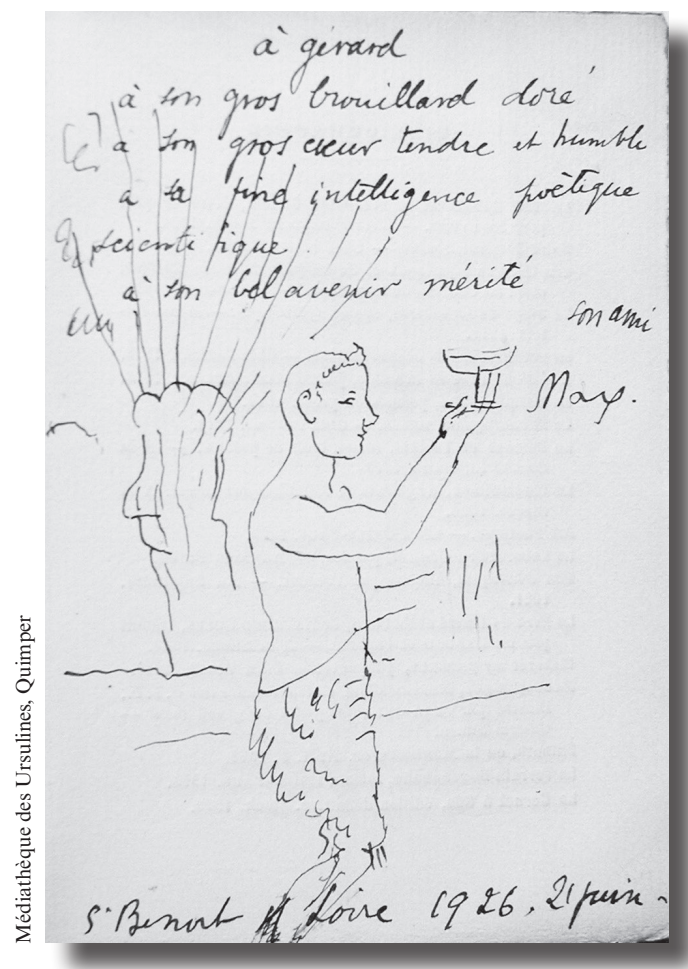

Le Cornet à dés, éd. de 1923, ex. $\mathrm{n}^{\circ} 8$ sur Hollande, dédicace à Gérard Magistry. au-dessus des vulgarités de la terre. La distinction de ses goûts lui tiendra lieu/de sens moral, mais quelles chutes! Colères inattendues! perte de cet équilibre radieux/gros rires, bouffonneries dont il faudra se/garder, violences même et trahisons... / Ne rendez pas malheureux des femmes qui vous entourent. Appliquez votre intuition à deviner le mal involontaire que vous causez/tournez en charité cette naturelle tendresse et, / vous entourant de bien, vous attirerez la chance. Vos dons d'acteur vous serviront à cette charité qui vous sauvera (et qui est si bien définie par st François de Sales) "soyez vendeur en achetant let achetez en vendant ». Comme vous êtes un parfait gentleman //

Ceci vous sera facile/Rassemblez votre dualité/Descendez vos multiples idées dans le cœur / Pour devenir un convaincu, ô beau joueur/Et laissez-moi m'incliner devant votre/matière décidément humaine, généreuse, libérale et progressive. I Max Jacob

\section{À Maurice Sachs ${ }^{51}$}

en souvenir des grands raids / de 1926, des grands raids / que nous continuons au travers / du monde sans fin. / Max Jacob / à mon fils Maurice Sachs 


\section{ÉDITION ORDINAIRE}

\section{À Marcel et Marguerite Béalu ${ }^{52}$}

à Marcel et à Marguerite

\section{À Madame Lionel Floch ${ }^{53}$}

à Madame Lionel Floch/respectueusement amicalement/Max Jacob/1933/le 16 septembre/anniversaire de Marie-Lionèle/Inédit/Sur la plage de vase/habitent des oiseaux/échassiers gris de plumagelbordés de jaune : ils / connaissent mes poèmes / du Cornet/à dés et les / chantent dans leurs / grands gros becs. / Max Jacob / Sous une grotte bleu du ciel/ habite une jonque de pierreries / qui est un crocodile.

\section{À Louis Guillaume ${ }^{54}$}

au noble Louis Guillaume / avec mon immense / affection/ Max Jacob 41

\section{À Mademoiselle Héléna Guillaumin ${ }^{55}$}

À Mademoiselle Héléna Guillaumin/en lui conseillant d'enlever/son $\mathrm{H}$ pour donner à son nom/sa pure signification/en la félicitant de sa grâcelet de sa patience au travail. / Avec mes hommages / Max Jacob / 40

\section{À Valéry Larbaud ${ }^{56}$}

à Valery Larbaud, ce livre n'a plus qu'une robe elle est voyante mais pauvre. C'est elle qui lui va mieux. Puissiez-vous l'aimez ainsi. Avec la vive admiration amie de Max Jacob.

\section{À Philippe de Lavastine ${ }^{57}$}

Fais un retour vers Dieu Philippe, qui te fera faire des progrès énormes.

\section{À Pierre Le Lann ${ }^{58}$}

à mon caissier/à mon impressario/à mon ami nouveau/et ancien/Pierre Le Lann/Max Jacob 35/Souvenir de ma conférencelà Angers/22 mai.

\section{À Robert Leplanquois ${ }^{59}$}

à Robert Leplanquois, son futur ami, Max Jacob, [19]28.

\section{À André Sauvage ${ }^{60}$}

à mon ami André/J'entendais ta voix avant/de l'entendre/J'avais vu tes yeux/dans mon propre miroir/J'ai connu ta pensée en/témoignant de la mienne / tu as lu mes livres / j'ai deviné les tiens / Max 


\section{À Marguerite et Roger Toulouse $\mathrm{e}^{61}$}

Puisque je suis sorcier, paraît-il, je vous prédis une énorme gloire la fortune et le bonheur aimez-moi tous les deux comme je vous aime/Max Jacob

\section{À Jean Tuset ${ }^{62}$}

Arbre, bel arbre de ma vie/poussez vos branches à tous mes/horizons / feuillages de ma fantaisie/poussez poussez jusqu'au plafond/M. J.//Je suis né le 12 et non pas/le 11/À bon entendeur salut/Donc salut à vous/ami docteur lettré votre Max Jacob//Inédits / Le jeune cheval hennissait. Il/montrait les dents et disait / au garçon d'écurie qui le / soignait: / "Vous me faites souffrir ! / La vieille jument regardait/son soigneur ou saigneur / d'un xil triste et ne disait/rien.

\section{À un dédicataire non identifié ${ }^{63}$}

Ah! tu ne l'a pas !/ tu ne l'as pas / la première édition, c'est rare. / Marquons le pas/en attendant le trépas/de monsieur Gallimard/son successeur le rééditera / si ce n'est pas trop tard/ Max Jacob 36

Sous son portrait par Picasso, le poète a écrit

Ce portrait ne m'a jamais/ressemblé de loin ni de près/c'est un dessin de café/ ramassé par je ne sais qui.

\section{ÉDITIONS NON IDENTIFIÉES}

\section{À Louis Guillaume ${ }^{64}$}

Au très profond, très sensible gentilhomme Louis Guillaume en union avec le granit gris et la sombre verdure bretonne.

\section{À Henry Lasserre ${ }^{65}$}

Puisse ce Cornet verser un peu de mon amitié : la donner entière serait impossible.

\section{ÉDITIONS ET DÉDICATAIRES NON IDENTIFIÉS ${ }^{66}$}

Je couds avec les fils de mes souffrances le fil de ma vie. 1928 


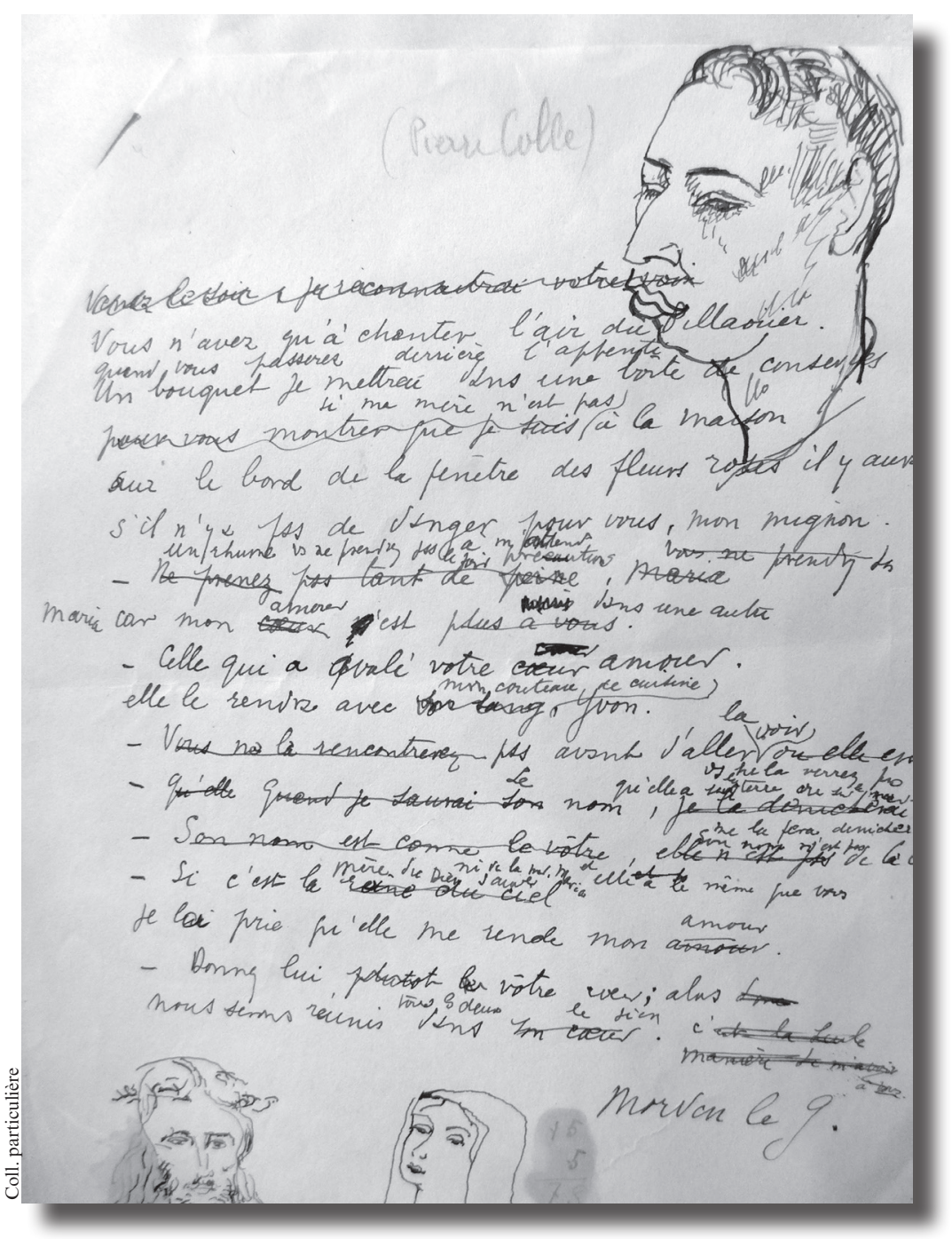

Max Jacob, Le Cornet à dés, exemplaire personnel de l'auteur offert à Monny de Boully en 1942. Brouillon manuscrit autographe à l'encre noir truffant l'exemplaire et comportant des fragments poétiques signé « Morven le G. » en page de faux titre du recueil, dessins à la plume : en haut à droite portrait de Pierre Colle (légendé par une tierce main au crayon graphite) en bas tête de saint et de la Vierge (dessins rognés à la reliure). 


\section{NOTES}

1 Lettre inédite de Paul Éluard à Max Jacob, 21 mars 1942 (MO-Ms 2305). Éluard présentera à Jacob plusieurs de ses livres à dédicacer. Filibuth, La Côte sont aujourd'hui propriété de la MO.

2 « À André Salmon/poète et précurseur des poètes modernes,/mon admiré et respecté ami,/Max Jacob » voir DURAND GUIZIOU Marie-Claire, " À propos d'une dédicace poétique de Max Jacob à André Salmon », Philologica canariensia, nº 8-9, 2002-2003, p. 75-85.

3 Voir PONGE Francis, Album amicorum: Gallimard, Les Cahiers de la NRF, textes réunis par Armande Ponge, postface de Gérard Farasse, 2009, p. 234.

4 Sur ces déplacements de fonction voir GENETTE Gérard, Seuils, éd. du Seuil, coll. Poétique, 1987, p. 116 et PUECH Jean-Benoît et COURATIER Jacky, « Dédicaces d'exemplaires », Poétique, nº 69, février 1987, p. 61-83.

5 Conte paru dans Les Lectures de la Semaine en mars 1904, voir CMJ, n 15/16, 2015, p. 31-59.

$6 \quad$ POUGY Liane de, Mes Cahiers bleus, Plon, 1977, p. 194.

7 Bibliothèque d'un amateur, Surréalisme, dadaïsme, cubisme, livres \& manuscrits, dessins \& illustrations, reliures, Hôtel Drouot, 23-24 mars 1981, lot 198. Le recueil porte également sous l'eau-forte de Picasso la signature au crayon de Max Jacob. Une lettre de Jacob à Léonce Rosenberg ainsi qu'une carte postale au même datée du 31 mars 1920 ont également été montés en tête par le relieur lors de la mise en œuvre de l'habillage (1953). La «fascinante reliure surréaliste mosaïquée en relief » de Paul Bonet est reproduite en regard du lot 165 : elle représente sur fond de box vert une main ouverte de couleur rouge sur laquelle sont incrustées des cartes de format carré ornées d'étoiles les unes dans un cercle noir, l'autre dorée. Une troisième carte porte six étoiles incrustées dans des cercles noires. Des formes oblongues de cuir mosaïquées ornent les plats. Nous remercions Mme Mille (Hôtel Drouot) et Mme Anne Verdure-Mary (BnF) de leur aide précieuse pour retrouver ce précieux catalogue qui présentait de très nombreux lots issus de la bibliothèque du Colonel Sicklès relatifs à Max Jacob.

8 Cet aphorisme est publié dans Visions infernales (1924; O., 648). Voir infra l'article « Histoire brève d'un recueil » et $C A T$., 155 .

9 Nous publions ici le texte de cet envoi tel qu'il se présente au manuscrit. Exemplaire personnel de Paul Bonet conservé à la BnF, Réserve des livres rares. Cet envoi a été publié pour la première fois en 1945 et repris depuis dans toutes les éditions ultérieures du recueil $(O ., 428)$. Voir supra l'article de Jean-Marc Pontier et Patricia Sustrac.

10 Voir infra « Bibliographie du poème en prose ».

11 Coll. particulière.

12 Envoi sur page de faux titre en pleine page représentant un trio de jeunes muses dansantes, Sotheby's, vente du 15 décembre 2015, lot 101. Adolphe Basler (1878-1949) était marchand et critique d'art et fréquentait les milieux artistiques des années vingt.

13 BOULOUQUE Claudine, BOURDOIS Claude, Max Jacob dans ses livres : Les Amis de la Reliure originale, catalogue de l'exposition organisée à la BHVP en 1994, p. 16-18.

14 Pour ce recueil et le suivant voir infra « Bibliographie du poème en prose ».

15 Don du 13 février 1940.

16 Coll. particulière. L'ouvrage comporte de nombreuses marginalia, dessins et esquisses.

17 Coll. du libraire Jean-Claude Vrain.

18 Souscripteur du Cornet non répertorié dans la liste donnée à Jean Paulhan. Fonds Pollès, Rennes.

19 BnF, RES-Z LE MASLE-199. L'envoi est porté sur l'avant-titre. Le recueil est enrichi d'ajouts 
manuscrits représentant des croix accolant de nombreux poèmes. Page 65, Cocteau a écrit au crayon graphite en marge des aphorismes "Le toit, c'est quatre, quatre, quatre... ", « Calembours grecs des oracles et de tables tournantes. » Page 117 en marge du poème « Mœurs littéraires » les initiales : « J. J. R. »

20 Léon Deffoux (1881-1945), poète, historiographe de l'Académie Goncourt.

21 Collection Nathalie Desse-Besset. Voir illustration.

22 Auction Art, vente du 11 avril 1988, lot 290.

23 ÉMIÉ Louis, Dialogues avec Max Jacob, Bordeaux : Le Festin, 1994, p. 23. Ex. mis en vente par la librairie Walden, 11 janvier 2017. L'ex. porte en dernière page la signature du dédicataire et la date : « $1920 »$.

24 MO, Rés H 12121. S'agit-il de Léon Eyrolles (1861-1945) le fondateur de la librairie éponyme?

25 Max Jacob venait de rencontrer le jeune George Gabory (1899-1978) et avait entrepris de le convertir. Poète et romancier il a écrit un recueil de souvenirs, GABORY Georges, Apollinaire, Max Jacob, Gide, Malraux et $C^{\circ}$ : Jean-Michel Place, 1988 (la dédicace est citée p. 24).

26 Livres anciens et modernes, succession de F. J. Gould, 24 juin 1984, Monte Carlo, lot $n^{\circ} 95$.

27 Coll. Alain Segal. Jean Marchat (1902-1966) était sociétaire de la Comédie-Française. Il était un des familiers de l'Hôtel Nollet où Jacob avait résidé à son retour à Paris.

28 Coll. particulière.

29 BOULOUQUE Claudine, BOURDOIS Claude, Max Jacob dans ses livres, op. cit., np.

30 Jean Hugo (1899-1984) est l'illustrateur du Cornet paru en 1948 chez Gallimard. Cf. supra l'article de Cécile Coutin.

31 Exemplaire enrichi d'un dessin à la plume, vente Rossini, 15 oct. 2013, lot 160. Nous n'avons pas identifié ce personnage dont la fille était la filleule de Jacob.

32 Coll. particulière.

33 Voir infra « Bibliographie du poème en prose ».

34 Vente Alde oct. 2014, lot 220. La Bibliothèque de Pierre Bergé, troisième vente, 28 juin 2017, Drouot, lot 724 .

35 Jacob avait écrit : «à Raymond Radiguet, poète mondial plutôt que national et qui ressemble à Musset "comme un frère" le vrai. Son ami déjà. Max Jacob, février 1919 ».

36 BLJD fds Max Jacob.

37 Coll. particulière.

38 Voir supra l'article « Brève histoire d'un recueil ».

39 Voir infra « Bibliographie du poème en prose ».

40 MJMB, p. 52.

41 Don du 28 juillet 1939. MUQ, FMJ9.

42 eBay (avril 2016). Le recueil présente un dessin autographe, encre et crayons de couleurs sur la couverture.

43 Herman de Guchtenaere dit Paul Méral (1895-1946), poète belge d'expression française, collaborateur de La Revue européenne. Coll. particulière.

44 Vente Ferraton, 23 sept. 2006, lot 142.

45 Voir infra « Bibliographie du poème en prose ».

46 Ouvrage enrichi de cinquante dessins à l'aquarelle ou lavis de Max Jacob. La Bibliothèque de Pierre Bergé, troisième vente, op. cit., lot 725.

47 Ex. sur Hollande $n^{\circ}$ 4, Musée Picasso, MP 2003-15. L'exemplaire est relié in 16 1/2, vélin à la bradel, avec plats recouverts de papier doré dont le premier porte - dans le coin supérieur droit - la mention « Jean » accompagnée d'une étoile à cinq branches dont Cocteau orne souvent ses 
manuscrits. La reliure réunit l'édition dite « définitive » du Cornet parue en 1923 chez Stock et douze feuillets de papier vergé sur lesquels sont collés 15 photographies prises par Jean Cocteau le 12 août 1916. Nous remercions Jeanne Sudour de son aide.

48 Ex. $n^{\circ} 16 . \mathrm{BnF}$, Réserve des Livres rares (ex. encore non coté). Un dessin original à l'encre et au crayon, signé par Max Jacob, est également monté entre la page de justification de tirage et le feuillet blanc qui suit (don Jacqueline Trutat). Nous remercions vivement M. Chatelain, Conservateur-directeur de la Réserve rare de la BnF.

49 Ex. $n^{\circ}$ 8. La dédicace est accompagnée d'un dessin. MU, FMJ A 346.

50 La dédicace est écrite sur deux exemplaires, le texte se faisant suite (http://www.priv-artcollection.com/artistes-Max_Jacob-Le_Cornet_a_Des-6-49-211.html). Premier ex. sur Hollande ( $\left.\mathrm{n}^{\circ} 17\right)$, le second est une édition ordinaire (1923).

51 Ex. sur Hollande ${ }^{\circ} 10$, reliure de Pierre-Lucien Martin, demi-maroquin vieux rouge à coins, dos à nerfs pincés, tête dorée, couverture, étui-boîte postérieur de papier tourniquet doublé de moire noire. La dédicace est portée sur la page de faux-titre. Vente Tajan, Bibliothèque de Claude Morillon, 20 sept. 2016, lot 149. Nous remercions le libraire Patrick Hatchuel de nous avoir communiqué la notice de son catalogue lors de la vente initiale du recueil en 1989.

52 BnF, NAF 28312 (99), recueil orné d'un croquis.

53 Coll. particulière.

54 Carnets de l'Association Louis Guillaume, n² 29, 2004, p. 79.

55 Fiancée de Marcel Métivier, jeune admirateur, alors en correspondance avec le poète. Coll. particulière.

56 MOUSLI Béatrice, Valéry Larbaud, Flammarion, coll. Grandes Biographies, 1998.

57 Librairie Le Feu Follet, Paris.

58 Coll. particulière.

59 Ouvrage enrichi d'un dessin, Abebooks, juin 2015.

60 MO sans cote.

61 MO, RES HL, p 455.

62 TUSET Jean, Les Amis d'Augustin Tuset, Max Jacob, Jean Moulin, Céline à Quimper, Lanrodec : Scripta, 2003, p. 86.

63 CAT., 238.

64 Coll. particulière.

65 LASSERRE Henry, « Deux jours chez Max Jacob », Le Cinéma des Muses, 27 avril 1963, p. 671.

66 JACOB Max, Lettres [à Théophile Briant et Conrad Moricand] 1920-1941, correspondance établie par Stanley J. Collier, Oxford : Basil Blackwell, 1966, p. 13. 